\title{
Major interventions are associated with survival of out of hospital cardiac arrest patients - a population based survey
}

Yi-Chuan Chen ${ }^{1,2}$, Ming-Szu Hung ${ }^{3,4}$, Chia-Hao Chang ${ }^{2,5,}$, Chia-Yen Liu ${ }^{6}$, Pau-Chung Chen ${ }^{7}$, Cheng-Ting Hsiao ${ }^{1,4}$, Yao-Hsu Yang ${ }^{6,8,9}$

${ }^{1}$ Department of Emergency Medicine, Chang Gung Memorial Hospital, Puzih City, Taiwan

${ }^{2}$ Department of Nursing, Chang Gung University of Science and Technology, Chiayi, Taiwan

${ }^{3}$ Division of Thoracic Oncology, Department of Pulmonary and Critical Care Medicine, Chang Gung Memorial Hospital, Puzih City, Taiwan

${ }^{4}$ Chang Gung University College of Medicine, Gueishan Township, Taiwan

${ }^{5}$ College of Nursing \& the Chronic Diseases and Health Promotion Research Center, Chang Gung University of Science and Technology, Chiayi, Taiwan

${ }^{6}$ Health Information and Epidemiology Laboratory of Chang Gung Memorial Hospital, Chiayi, Taiwan

${ }^{7}$ Institute of Occupational Medicine and Industrial Hygiene, National Taiwan University College of Public Health, Taipei, Taiwan

${ }^{8}$ Department of Traditional Chinese Medicine, Chiayi Chang Gung Memorial Hospital, Chiayi, Taiwan

${ }^{9}$ School of Traditional Chinese Medicine, College of Medicine, Chang Gung University, Taoyuan, Taiwan

Corresponding author:

Yao-Hsu Yang

Department of Traditional Chinese Medicine

Chiayi Chang Gung Memorial Hospital

Chiayi, Taiwan, No.6, W. Sec. Jiapu Rd.

Puzih City, Chiayi County 613, Taiwan

E-mail:r95841012@ntu.edu.tw

\section{ABSTRACT}

Background. The overall survival rate of out-of-hospital cardiac arrest (OHCA) in Taiwan or even in the whole of Asia is relatively low. Major interventions, such as target temperature management (TTM), coronary artery angiography, and extracorporeal membrane oxygenation (ECMO), have been associated with better patient outcome. However, studies in Taiwan revealing evidence of the benefits of these interventions are limited.

Methods. A population-based study used an 8-year database to analyze overall survival and risk factors "among OHCA patients. All adult non-trauma OHCA patients were identified through diagnostic and procedure codes. Hospital survival and return of spontaneous circulation (ROSC) were primary and secondary outcomes. Logistic regression and Cox regression analyses were conducted.

Results. There was a relationship between major interventions (including TTM, coronary artery angiography, and ECMO) and better hospital survival. Age, income, major interventions, and acute myocardial infarction history were associated with hospital survival. The adjusted hazard ratios (HRs) were 0.406 (95\% CI, 0.295 to 0.558$), 1.109$ (95\% CI, 1.027 to 1.197 ), 1.075 (95\% CI, 1.002 to 1.154$), 1.097$ (95\% CI, 1.02 to 1.181 ) and $0.799(95 \%$ CI, 0.677 to 0.942 ) for patients with major interven- tions, age $\geq 50$, medium low and low income, middle income, and acute myocardial infarction history, respectively.

Conclusion. This population-based study in Taiwan revealed that older age $(\geq 50)$, medium low and low income were associated with a lower rate of survival. Major interventions, including TTM, coronary angiography, and ECMO, were related to better survival.

Key words: OHCA, ROSC, out-of-hospital cardiac arrest, target temperature management, ECMO

\section{INTRODUCTION}

Out-of-hospital cardiac arrest (OHCA) remains a leading cause of mortality and a substantial issue for public health worldwide. According to a statistical update from the American Heart Association, the incidence of OHCA in the United States is approximately 359,400 each year with an overall survival rate of $9.5 \%$. (1) Overall survival to hospital discharge rates are lower in the Asian population with rates ranging from $0.5 \%$ to $8.5 \%$. (2-4) The overall survival to hospital discharge in Taiwan ranges from $1.4 \%$ to $6 \%$. (5-7) To overcome the low survival rate, the concept of an out-of-hospital chain of survival has been established, including 1) early recognition of cardiac arrest and activation of the emergency medical service (EMS) system, 2) early cardiopulmonary resuscitation (CPR) with highlight on high quality chest compressions, 3) early defibrillation, 4) basic and advanced EMS, 5) advanced life support (ALS) and post-cardiac arrest care. (8)

Several interventions, such as extracorporeal membrane oxygenation (ECMO), $(9,10)$ target temperature management (TTM), $(11,12)$ and percutaneous coronary intervention, $(13,14)$ have been associated with favorable patient outcomes. In Taiwan, there is limited data on major interventions (TTM, coronary artery angiography, ECMO) and their influence on patient outcomes. The aim of our study was to assess the impact of patient demographic features, hospital characteristics, and major interventions on prognoses of OHCA.

\section{METHODS}

\section{Data Sources}

The National Health Insurance (NHI) program was conducted in Taiwan in 1995 and provides compulsory universal health insurance. It enrolls about $99 \%$ of the Taiwanese population and contracts with $97 \%$ of all the country's health care services. (15) For research purposes, a random sample of $1,000,000$ people who received 
health services from the NHI program was selected based on calendar-year 2005 reimbursement data. According to the Taiwan National Health Research Institute (NHRI), the random sample group is considered representative of the entire population since the group did not differ statistically from the whole cohort in age, sex, or health care costs. This random sample of 1,000,000 people was used as our study cohort. Information about admissions and outpatient visits, both of which included information on patient characteristics (sex, date of birth, date of admission, date of discharge, dates of visits) and up to five discharge diagnoses or three out-patient visit diagnoses (based on the International Classification of Diseases, Ninth Revision [ICD-9]), was collected. (16) We also gained information on patient prescriptions, including the names of prescribed drugs, dosage, duration, and total expenditure. Previously used for epidemiologic research, the NHRI database provided high quality information on prescription use, diagnoses, and hospitalizations (17) and this database had been previously used for OHCA research. $(18,19)$ The NHRI strictly followed confidentiality guidelines in accordance with personal electronic data protection regulations, making the $\mathrm{NHI}$ reimbursement data suitable for research. (15) In addition, this study was approved by Chang Gung Medical Foundation Institutional Review Board.

\section{Identification of Study Sample}

We carried out a population-based cohort study in which we identified all alive patients older than 18 years who suffered from out-of-hospital cardiac arrest (OHCA) (ICD-9 codes 427.5, 798.9, 798.1, 798.2) and were admitted to the emergency department (ED) between January 1, 2005 and December 31, 2012. Thus, we formed a study cohort with a total number of 6,815 individuals. Individuals with cardiac arrest announced death by EMS at the scene or without transportation to the hospital were not included because EMS records were not available in our NHI database. In Taiwan, prehospital emergency care is provided by emergency medical technicians (EMTs) in different fire bureaus. Most EMTs are EMT-II, but some cities have paramedics (EMT-Ps). Once a patient with cardiac arrest is identified, EMTs or EMT-Ps do not terminate resuscitation even when the patient had signed a do not resuscitate (DNR) consent or has signs of obvious death (rigor, mortis, lividity, decapitation etc.) unless the patient's family understands and requests to discontinue. Complete prehospital advanced life support (ALS) is performed only in some urban areas since the number of EMT-Ps is limited. On the basis of an evaluation in urban Taipei, ALS demand accounted for approximately $9-16 \%$ of total EMS calls, and the average response time was 4.1-4.9 minutes. In rural areas, the average response time was found to be longer, even exceeding 6.6 minutes. (20)

Two hundred and twenty-four (224) individuals with trauma, intoxication, submersion, and accidents (ICD-9 codes 800-999 and E800-999) were excluded. Those who did not receive any CPR, defibrillation, or endotracheal intubation during ED stay were also excluded (number $=1253$ ). All enrolled patients were followed up until December 31, 2013 (Figure1). The primary outcome was hospital survival, which was defined as survival to hospital discharge. The secondary outcome was return of spontaneous circulation (ROSC), which was defined in this study as sustained ROSC with hospital admission.

\section{Potential Covariates and Management}

We identified in a systematic way any comorbid conditions as potential confounders, defined by the following diagnoses recorded before January 1, 2005: angina (ICD-9 codes 413.0, 413.1, and 413.9), acute myocardial infarction (ICD-9 codes $410.70,410.71,410.72,410.80,410.81$, $410.82,410.90,410.91,410.92)$, coronary artery disease (ICD-9 codes 410.x-414.x), diabetes (ICD-9 codes250.0-250.3, 250.8, 250.9), malignancies (ICD-9 codes140.x172.x, 174.x-195.8, 200.x-208.x, 238.6), heart failure (ICD-9 codes398.91, 402.01, 402.11, 402.91, 404.01, 404.03, 404.11, $404.13, \quad 404.91, \quad 404.93, \quad 425.4-425.9$, 428.x), cerebrovascular disease (ICD-9 codes362.34, 430.x-438.x), and the Charlson Comorbidity Index (CCI). The CCI is a scoring system that strengthens important concomitant diseases and has been validated for studies that employ ICD-9 data. (18)

We also considered socio-demographic characteristics (age, sex, level of urbanization, level of hospital, and level of monthly income) in the modeling. Level of hospital is classified as either medical center or non-medical center. (21) Urbanization levels in Taiwan are divided into seven groups according to the Taiwan National Health Research Institute publications. (22) Group 1 is called highly urbanized area, group 2 is moderately urbanized area, group 3 is emerging area, group 4 is gen-

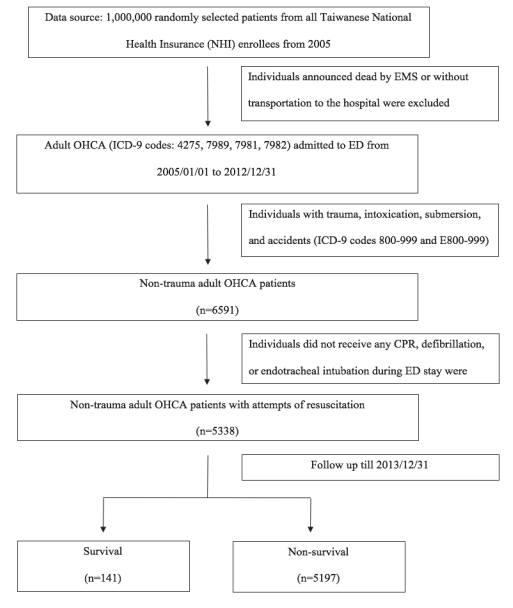

Figure 1. Flow chart of identification of study sample and follow up.

$\mathrm{CPR}$, cardiopulmonary resuscitation; ED, emergency department; EMS, emergency medical system; ICD-9, International Classification of Diseases, Ninth Revision; OHCA, out of hospital cardiac arres.

eral rural area, group 5 is aging area, group 6 is agricultural area, and group 7 is remote area. (22) In our study, groups 4, 5, 6, and 7 are merged into a single group, called general rural area. Thus, urbanization levels in our report are classified as highly urbanized area, moderately urbanized area, emerging area and general rural area. Individual monthly income in New Taiwan dollars (NTD) is divided into four groups: $0,1-15,840,15,841-25000$ and $\geq 25,001$. According to the Ministry of Health and Welfare of Taiwan, individual monthly income less than 150 percent of the poverty level amount is defined as low income. (23) Thus, we classified these four groups as follows: no income (0), medium low and low income (1-15,840), middle income (15,841-25000), high income ( $\geq 25,001)$.

For the survey of factors associated with hospital survival, major interventions, which were composed of three managements: TTM, coronary artery angiography, and ECMO, were identified during the hospital stay.

\section{Statistical Analyses}

We used the Kaplan-Meier method to analyze the survival of patients with and without major interventions, and the log-rank test was performed to examine the differences in mortality. Cox proportional hazard models were used to compute the hazard ratios (HRs) for mortality of OHCA patients and logistic regression model was 
applied for risk analysis of ROSC, accompanying 95\% confidence interval (CI) after adjustment for the variables mentioned. Two-tailed $\mathrm{P}<0.05$ was considered significant. All of these analyses were conducted using SAS statistical software (version 9.4; SAS Institute, Cary, NC).

\section{RESULTS}

Figure 1 illustrates the selection process of study participants. A total of 5,338 OHCA patients met inclusion and exclusion criteria for this study. Of these patients, $12.2 \%$ (652) were admitted to hospital and $2.64 \%$ (141) survived to hospital discharge. Table 1 lists the demographic characteristics of all OHCA patients. Twenty-three (0.4\%) patients received TTM, 19 (0.36\%) received coronary artery angiography, 11 (0.2\%) received ECMO, and 48 (0.9\%) received major interventions. Cox regression model reveals that age, income, major interventions, and acute myocardial infarction history were associated with hospital survival after adjusting for gender, age, urbanized level, individual income, major interventions, level of hospital, Charlson comorbidity index, angina, acute myocardial infarction, any tumor, cerebrovascular disease, congestive heart failure, coronary artery disease, and diabetes (table 2). A logistic regression model demonstrates factors associated with ROSC, including age, income, and comorbidities with coronary artery disease and cerebrovascular disease after adjusting for every variable in Table 2 (table 3). Figure 2 illustrates the results of the Kaplan-Meier method for the cohort.

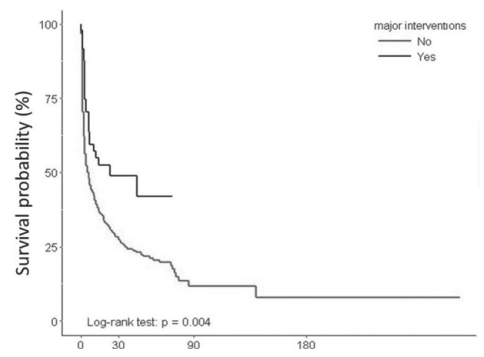

Figure 2. Kaplan-Meier estimates of survival of patients with and without major interventions ( $p=0.004)$.

Table 1. Demographic characteristics of OHCA patients

\begin{tabular}{|c|c|c|}
\hline \multirow[t]{2}{*}{ Variables } & \multicolumn{2}{|c|}{ OHCA $(\mathrm{N}=5338)$} \\
\hline & $\mathbf{n}$ & $\%$ \\
\hline \multicolumn{3}{|l|}{ Gender } \\
\hline Female & 1989 & 37.26 \\
\hline Male & 3349 & 62.74 \\
\hline \multicolumn{3}{|l|}{ Age } \\
\hline Age $<50$ & 927 & 17.37 \\
\hline Age $\geq 50$ & 4411 & 82.63 \\
\hline \multicolumn{3}{|l|}{ Urbanized level } \\
\hline highly urbanized area & 1323 & 24.78 \\
\hline moderately urbanized area & 2506 & 46.95 \\
\hline emerging area & 979 & 18.34 \\
\hline general rural area & 530 & 9.93 \\
\hline \multicolumn{3}{|l|}{ Individual income } \\
\hline No income & 1944 & 36.42 \\
\hline \multicolumn{3}{|l|}{ Medium low and Low } \\
\hline income & 1512 & 28.33 \\
\hline Middle income & 1518 & 28.44 \\
\hline High income & 364 & 6.82 \\
\hline \multicolumn{3}{|l|}{ ROSC } \\
\hline No & 4622 & 86.59 \\
\hline Yes & 716 & 13.41 \\
\hline OPD follow up & 64 & 1.2 \\
\hline Admission & 652 & 12.2 \\
\hline Major interventions & 48 & 0.9 \\
\hline No major interventions & 604 & 11.3 \\
\hline Survival & 141 & 2.64 \\
\hline Non-survival & 5197 & 97.36 \\
\hline \multicolumn{3}{|l|}{ Level of hospital } \\
\hline Medical center & 1373 & 25.72 \\
\hline Non-medical center & 3965 & 74.28 \\
\hline
\end{tabular}




\section{Covariates}

Charlson comorbidity index

\begin{tabular}{lll}
\hline 0 & 3171 & 59.4 \\
1 & 597 & 11.2 \\
$\geq 2$ & 1570 & 29.4 \\
\hline Angina & 115 & 2.15 \\
\hline Any tumor & 367 & 6.88 \\
\hline Acute myocardial infarction & 176 & 3.3 \\
\hline Cerebrovascular disease & 451 & 8.45 \\
\hline Congestive heart failure & 471 & 8.82 \\
\hline Coronary artery disease & 365 & 6.84 \\
\hline Diabetes & 296 & 5.55 \\
\hline
\end{tabular}

OHCA, out of hospital cardiac arrest; OPD, outpatient department; ROSC, return of spontaneous circulation.

Table 2. Cox regression analysis for hospital survival of all OHCA patients

\begin{tabular}{|c|c|c|c|c|c|c|c|c|}
\hline \multirow{3}{*}{$\begin{array}{l}\text { Variables } \\
\text { Gender }\end{array}$} & \multicolumn{4}{|c|}{ Crude hazard ratio } & \multicolumn{4}{|c|}{ Adjusted hazard ratio } \\
\hline & \multicolumn{3}{|c|}{ hazard ratio $95 \% \mathrm{CI}$} & \multirow[t]{2}{*}{$\mathbf{P}$} & \multicolumn{3}{|c|}{ hazard ratio $95 \% \mathrm{CI}$} & \multirow[t]{2}{*}{$\mathbf{P}$} \\
\hline & & & & & & & & \\
\hline Female & 1 & & & & 1 & & & \\
\hline Male & 1.017 & 0.962 & 1.076 & 0.5466 & 1.017 & 0.96 & 1.077 & 0.5748 \\
\hline \multicolumn{9}{|l|}{ Age } \\
\hline$<50$ & 1 & & & & 1 & & & \\
\hline$\geq 50$ & 1.094 & 1.018 & 1.177 & $0.0149^{*}$ & 1.109 & 1.027 & 1.197 & $0.0082^{*}$ \\
\hline \multicolumn{9}{|l|}{ Urbanized level } \\
\hline highly urbanized area & 0.919 & 0.83 & 1.017 & 0.1037 & 0.982 & 0.882 & 1.094 & 0.7418 \\
\hline moderately urbanized area & 0.934 & 0.849 & 1.026 & 0.1553 & 0.972 & 0.882 & 1.072 & 0.5748 \\
\hline emerging area & 0.987 & 0.887 & 1.098 & 0.8041 & 1 & 0.898 & 1.112 & 0.9933 \\
\hline general rural area & 1 & & & & 1 & & & \\
\hline \multicolumn{9}{|l|}{ Individual income } \\
\hline No income & 1 & & & & 1 & & & \\
\hline Medium low and Low income & 1.073 & 1.002 & 1.149 & $0.0436^{*}$ & 1.075 & 1.002 & 1.154 & $0.0427^{\star}$ \\
\hline Middle income & 1.104 & 1.031 & 1.182 & $0.0046^{*}$ & 1.097 & 1.02 & 1.181 & $0.0125^{*}$ \\
\hline High income & 1.002 & 0.894 & 1.123 & 0.9704 & 1.032 & 0.918 & 1.16 & 0.5965 \\
\hline \multicolumn{9}{|l|}{ Interventions } \\
\hline no major interventions & 1 & & & & & & & \\
\hline major interventions & 0.38 & 0.277 & 0.522 & $<.0001^{\star}$ & 0.406 & 0.295 & 0.558 & $<.0001^{*}$ \\
\hline \multicolumn{9}{|l|}{ Level of hospital } \\
\hline Non-Medical center & 1 & & & & 1 & & & \\
\hline Medical center & 0.908 & 0.853 & 0.966 & $0.0025^{\star}$ & 0.938 & 0.88 & 1.001 & 0.0537 \\
\hline \multicolumn{9}{|l|}{ Covariates } \\
\hline \multicolumn{9}{|l|}{ Charlson comorbidity index } \\
\hline 0 & 1 & & & & 1 & & & \\
\hline 1 & 1.019 & 0.938 & 1.106 & 0.6601 & 0.995 & 0.916 & 1.082 & 0.9139 \\
\hline$\geq 2$ & 1.04 & 0.98 & 1.104 & 0.1942 & 1.031 & 0.969 & 1.098 & 0.3366 \\
\hline Angina & 1.018 & 0.845 & 1.227 & 0.8472 & 1.093 & 0.902 & 1.325 & 0.3629 \\
\hline Acute myocardial infarction & 0.758 & 0.648 & 0.886 & $0.0005^{*}$ & 0.799 & 0.677 & 0.942 & $0.0077^{*}$ \\
\hline Any tumor & 1.014 & 0.911 & 1.127 & 0.8052 & 0.991 & 0.889 & 1.103 & 0.8636 \\
\hline Cerebrovascular disease & 0.957 & 0.868 & 1.054 & 0.372 & 0.946 & 0.857 & 1.044 & 0.2682 \\
\hline Congestive heart failure & 0.916 & 0.832 & 1.008 & 0.0723 & 0.936 & 0.847 & 1.034 & 0.1915 \\
\hline
\end{tabular}




\begin{tabular}{llllllllll}
\hline Coronary artery disease & 0.846 & 0.758 & 0.945 & 0.0029 & 0.896 & 0.796 & 1.008 & 0.067 \\
\hline Diabetes & 0.949 & 0.841 & 1.071 & 0.3988 & 0.924 & 0.732 & 1.167 & 0.507 \\
\hline
\end{tabular}

OHCA, out of hospital cardiac arrest.

Table 3. Logistic regression analysis for return of spontaneous circulation (ROSC)

\begin{tabular}{|c|c|c|c|c|c|c|c|c|}
\hline \multirow[t]{2}{*}{ Variables } & \multicolumn{4}{|c|}{ Crude odds ratio } & \multicolumn{4}{|c|}{ Adjusted odds ratio } \\
\hline & odds ratio & $95 \% \mathrm{CI}$ & & $\mathbf{P}$ & odds ratio & 95\% CI & & $\mathbf{P}$ \\
\hline \multicolumn{9}{|l|}{ Gender } \\
\hline Female & 1 & & & & 1 & & & \\
\hline Male & 1.195 & 1.018 & 1.403 & 0.0296 & 1.179 & 0.996 & 1.396 & 0.0559 \\
\hline \multicolumn{9}{|l|}{ Age } \\
\hline$<50$ & 1 & & & & 1 & & & \\
\hline$\geq 50$ & 1.209 & 0.991 & 1.476 & 0.0614 & 1.505 & 1.213 & 1.867 & $0.0002^{*}$ \\
\hline \multicolumn{9}{|l|}{ Urbanized level } \\
\hline highly urbanized area & 0.736 & 0.544 & 0.995 & 0.0466 & 0.936 & 0.677 & 1.294 & 0.6881 \\
\hline moderately urbanized area & 0.868 & 0.652 & 1.157 & 0.3348 & 1.024 & 0.758 & 1.385 & 0.8757 \\
\hline emerging area & 1.055 & 0.759 & 1.466 & 0.7503 & 1.112 & 0.796 & 1.555 & 0.5325 \\
\hline general rural area & 1 & & & & 1 & & & \\
\hline \multicolumn{9}{|l|}{ Individual income } \\
\hline No income & 1 & & & & 1 & & & \\
\hline Medium low and Low income & 1.209 & 0.997 & 1.466 & 0.0531 & 1.199 & 0.979 & 1.468 & 0.079 \\
\hline Middle income & 1.489 & 1.217 & 1.822 & 0.0001 & 1.412 & 1.134 & 1.759 & $0.002^{*}$ \\
\hline High income & 1.384 & 0.984 & 1.946 & 0.0619 & 1.466 & 1.026 & 2.094 & $0.0356^{*}$ \\
\hline \multicolumn{9}{|l|}{ Level of hospital } \\
\hline Non-Medical center & 1 & & & & 1 & & & \\
\hline Medical center & 0.668 & 0.564 & 0.792 & $<.0001$ & 0.701 & 0.586 & 0.838 & 0.0001 \\
\hline \multicolumn{9}{|l|}{ Covariates } \\
\hline \multicolumn{9}{|l|}{ Charlson comorbidity index } \\
\hline 0 & 1 & & & & 1 & & & \\
\hline 1 & 0.975 & 0.771 & 1.233 & 0.8323 & 0.92 & 0.723 & 1.173 & 0.5024 \\
\hline$\geq 2$ & 1.026 & 0.862 & 1.22 & 0.7736 & 1.142 & 0.947 & 1.378 & 0.1655 \\
\hline Angina & 1.337 & 0.731 & 2.443 & 0.3455 & 2.139 & 1.126 & 4.063 & $0.0201^{*}$ \\
\hline Acute myocardial infarction & 0.267 & 0.194 & 0.367 & $<.0001$ & 0.333 & 0.232 & 0.478 & $<.0001^{\star}$ \\
\hline Any tumor & 0.813 & 0.607 & 1.089 & 0.1643 & 0.759 & 0.561 & 1.027 & 0.0737 \\
\hline Cerebrovascular disease & 0.521 & 0.411 & 0.662 & $<.0001$ & 0.496 & 0.387 & 0.636 & $<.0001^{\star}$ \\
\hline Congestive heart failure & 0.522 & 0.413 & 0.661 & $<.0001$ & 0.608 & 0.47 & 0.788 & $0.0002^{*}$ \\
\hline Coronary artery disease & 0.463 & 0.359 & 0.597 & $<.0001$ & 0.605 & 0.451 & 0.812 & $0.0008^{\star}$ \\
\hline Diabetes & 0.831 & 0.599 & 1.154 & 0.2689 & 0.949 & 0.461 & 1.954 & 0.8864 \\
\hline
\end{tabular}

\section{DISCUSSION}

There were two main findings in this study. First, older age $(\geq 50)$, medium low and low income were associated with a lower rate of hospital survival. Second, major interventions (including TTM, coronary artery angiography, and ECMO) were associated with a higher rate of in-hospital survival. Consistent with other reports, $(24,25)$ the incidence of OHCA in men was higher than in women, and men were prone to have a lower rate of ROSC than women in our investigation. The possible reasons might be the effect of estrogen and progesterone on reduction of cardiac arrest risk in women and higher prevalence of lifestyle and cardiovascular risk factors in men. (26) However, the effect of gender on hospital survival of OHCA was not significant, as previous studies have described. $(24,25)$
According to a recent retrospective survey in Sweden, there was no association between urbanization and outcomes after OHCA, consistent with our discovery. (27) In contrast with urbanization, higher level of hospital care, such as in a medical center, was associated with successful ROSC in our report. Several studies have identified in-hospital factors associated with improved mortality after successful resuscitation from OHCA in patients ad- 
mitted to different hospitals with different levels of care. $(28,29)$ Level of hospital care has been suggested as an important factor for the difference in mortality of OHCA patients. One Denmark study demonstrated that a tertiary center had improved survival than other hospitals, and another Australia report declared that hospitals, especially those with 24-h cardiac intervention services, were associated with better outcomes in OHCA setting. $(29,30)$ According to data from the Ministry of Health and Welfare of Taiwan, all medical centers are capable of providing 24-h, 7 days per week emergency cardiac catheterization services for acute ST elevation myocardial infarction patients and cardiac surgeon consultation. (21)

In our study, medium low and low income was associated with a lower rate of hospital survival. Previous studies have revealed that persons of lower socioeconomic status (SES) have been found to have a greater load of risk factors for cardiovascular disease (31) and less knowledge of risk factors for cardiovascular disease. (32) One report, in a Taiwan metropolitan region, showed that areas with low SES were associated with worse patient outcomes following OHCA. (33) Lower rates of witnessed arrest and bystander cardiopulmonary resuscitation and higher incidence of cardiovascular disease were possible contributors to unfavorable outcomes in the low SES group after cardiac arrest. (34)

Several randomized trials have revealed that TTM is effective in the improvement of both neurological function and survival. $(11,12,35)$ Two trials focusing on OHCA with an initial shockable rhythm had demonstrated the association between TTM and good neurologic outcome $(11,12)$ and survival to hospital discharge. (11) Postarrest coronary artery angiography had influence on the increase of hospital survival and good neurologic function. (36-38) The benefit of emergency cardiac catheterization, compared with catheterization later during hospital stay or no intervention, has been documented among post-arrest patients, either with evidence of ST elevation on electrocardiography (ECG) $(36,37)$ or with no ST elevation on ECG. $(38,39)$

The benefit of ECMO on survival and neurological function in a selected patient group following OHCA had also been documented. $(9,10)$ Though there was a relatively small number of major interventions in our study, patients with TTM, or coronary artery angiography, or ECMO had higher hospital survival, in agreement with the results of many reports.

Limitations

There were several potential limitations of this study. First, the data was obtained from an administrative database, which has been shown in the past to have inaccuracies when used for cardiac arrest research. (40) To overcome this, procedures and drugs administrated during resuscitation were another key element in data selection in addition to ICD-9 diagnosis code. Only those with attempts in resuscitation were enrolled in the analysis.

Second, several unmeasured confounders, which were related to pre-hospital factors and the emergency medical service(EMS) system, including place of occurrence, witnessed collapse, bystander-initiated CPR, EMS response time, initial arrest rhythm, prehospital defibrillation, airway management, and drug administration, were not available in our database. Third, we were unable to document non-sustained ROSC but merely those sustained ROCS with survival to hospital admission or survival to outpatient follow up. Fourth, neurologic functional status was not available in our database; hence, the effect of major interventions on neurologic outcome could not be evaluated. Another concern of our report was that the numbers of patients treated with TTM and angiography were very low. One probable explanation is the treating physician's selection bias for who would receive the treatments. Another probable explanation is that some materials for TTM and angiography were not covered by NHI and were relatively expensive, decreasing patients' willingness to receive the interventions.

\section{CONCLUSION}

This population-based study in Taiwan revealed that older age $(\geq 50)$, medium low and low income were associated with a lower rate of survival. Major interventions, including TTM, coronary angiography, and ECMO were related to better survival.

\section{ACKNOWLEDGEMENTS}

The authors would like to thank the Health Information and Epidemiology Laboratory of Chang Gung Memorial Hospital, Chia-yi Branc (CLRPG6G0041) for comments and assistance in data analysis. This study was supported by a grant from Chang Gung Memorial Hospital, Chiayi Branch, and was based on the National Health Insurance Research Database provided by the Central Bureau of National Health Insurance, the Department of Health, and the National Health Research Institutes. The interpretation and conclusions contained herein do not represent those of Bureau of National Health Insurance, Department of Health, or National Health Research Institutes.

\section{REFERENCES}

1. Cardiac Arrest Statistics. [Online]. [2015] [cited 2016 Feb 06]; Available from: URL: http://cpr.heart.org/AHAECC/CPRAndECC/ General/UCM_477263_Cardiac-Arrest-Statistics.jsp

2. Hasegawa K, Tsugawa Y, Camargo CA, Jr., Hiraide A, Brown DF. Regional variability in survival outcomes of out-of-hospital cardiac arrest: the All-Japan Utstein Registry. Resuscitation 2013;84(8):1099-107.

3. Kim JY, Shin SD, Ro YS, Song KJ, Lee EJ, Park CB, et al. Post-resuscitation care and outcomes of out-of-hospital cardiac arrest: a nationwide propensity score-matching analysis. Resuscitation 2013;84(8):1068-77.

4. Ong ME, Shin SD, De Souza NN, Tanaka H, Nishiuchi T, Song KJ, et al. Outcomes for out-of-hospital cardiac arrests across 7 countries in Asia: The Pan Asian Resuscitation Outcomes Study (PAROS). Resuscitation 2015;96:100-8.

5. Ma MH, Chiang WC, Ko PC, Huang JC, Lin CH, Wang HC, et al. Outcomes from out-of-hospital cardiac arrest in Metropolitan Taipei: does an advanced life support service make a difference? Resuscitation 2007;74(3):461-9.

6. Kuo CW, See LC, Tu HT, Chen JC. Adult out-of-hospital cardiac arrest based on chain of survival in Taoyuan County, northern Taiwan. J Emerg Med 2014;46(6):782-90.

7. Wang CY, Wang JY, Teng NC, Chao TT, Tsai SL, Chen CL, et al. The secular trends in the incidence rate and outcomes of out-ofhospital cardiac arrest in Taiwan--a nationwide population-based study. PloS one 2015;10(4):e0122675. 
8. Peberdy MA, Callaway CW, Neumar RW, Geocadin RG, Zimmerman JL, Donnino M, et al. Part 9: post-cardiac arrest care: 2010 American Heart Association Guidelines for Cardiopulmonary Resuscitation and Emergency Cardiovascular Care. Circulation 2010;122(18 Suppl 3):S768-86.

9. Maekawa K, Tanno K, Hase M, Mori K, Asai Y. Extracorporeal cardiopulmonary resuscitation for patients with out-of-hospital cardiac arrest of cardiac origin: a propensity-matched study and predictor analysis. Crit Care Med 2013;41(5):1186-96.

10. Sakamoto T, Morimura N, Nagao K, Asai Y, Yokota H, Nara S, et al. Extracorporeal cardiopulmonary resuscitation versus conventional cardiopulmonary resuscitation in adults with out-of-hospital cardiac arrest: a prospective observational study. Resuscitation 2014;85(6):762-8.

11. Hypothermia after Cardiac Arrest Study G. Mild therapeutic hypothermia to improve the neurologic outcome after cardiac arrest. N Engl J Med 2002;346(8):549-56.

12. Bernard SA, Gray TW, Buist MD, Jones BM, Silvester W, Gutteridge G, et al. Treatment of comatose survivors of out-of-hospital cardiac arrest with induced hypothermia. N Engl J Med 2002;346(8):557-63.

13. Spaulding CM, Joly LM, Rosenberg A, Monchi M, Weber SN, Dhainaut JF, et al. Immediate coronary angiography in survivors of out-of-hospital cardiac arrest. N Engl J Med 1997;336(23):1629-33.

14. Zanuttini D, Armellini I, Nucifora G, Carchietti E, Trillo G, Spedicato L, et al. Impact of emergency coronary angiography on inhospital outcome of unconscious survivors after out-of-hospital cardiac arrest. Am J Cardiol 2012;110(12):1723-8.

15. National Health Research Institutes: National Health Insurance Research Database. [Online]. 2016 [cited 2016 Feb 02]; Available from: URL: http://nhird.nhri.org.tw/date_01_en.html

16. Centers for Disease Control and Prevention: International Classification of Diseases, Ninth Revision (ICD-9) [Online]. 2016 [cited 2016 Feb 07]; Available from: URL: https://www.cdc.gov/nchs/icd/icd9.htm

17. Yang YH, Chen WC, Tsan YT, Chen MJ, Shih WT, Tsai YH, et al. Statin use and the risk of cirrhosis development in patients with hepatitis C virus infection. J Hepatol 2015;63(5):1111-7.

18. Chen JJ, Lee YK, Hou SW, Huang MY, Hsu CY, Su YC. End-tidal carbon dioxide monitoring may be associated with a higher possibility of return of spontaneous circulation during out-of-hospital cardiac arrest: a population-based study. Scand J Trauma Resusc Emerg Med 2015;23:104.

19. Dorji Harnod MH-MM, Wen Han Chang, Ray-E Chang, Chu-Hui Chang. Mortality Factors in Out-of-Hospital Cardiac Arrest Patients: A Nationwide Population-based Study in Taiwan. I Int J Gerontol 2013;7:216-20.

20. Chiang WC, Ko PC, Wang HC, Yang CW, Shih FY, Hsiung KH, et al. EMS in Taiwan: past, present, and future. Resuscitation 2009;80(1):9-13.

21. Ministry of Health and Welfare. [Online]. 2016 [cited 2016 June 1]; Available from: URL: http://dep.mohw.gov.tw/DOMA/cp-27107581-106.html

22. Chieh-Yu Liu Y-TH, Yi-Li Chuang, Yi-Ju Chen,Wen-Shun Weng, Jih-Shin Liu, Kung-Yee Liang. Incorporating development stratifiation of Taiwan townships into sampling design of large scale health interview survey. J Health Manag 2006;4(1):1-22.

23. Ministry of Health and Welfare: Laws and Regulations. [Online]. 2016 [cited 2016 June 1]; Available from: URL: https://www.mohw. gov.tw/cp-190-237-1.html

24. Bray JE, Stub D, Bernard S, Smith K. Exploring gender differences and the "oestrogen effect" in an Australian out-of-hospital cardiac arrest population. Resuscitation 2013;84(7):957-63.

25. Ng YY, Wah W, Liu N, Zhou SA, Ho AF, Pek PP, et al. Associations between gender and cardiac arrest outcomes in Pan-Asian out-ofhospital cardiac arrest patients. Resuscitation 2016;102:116-21.

26. McCullough LD, Hurn PD. Estrogen and ischemic neuroprotection: an integrated view. Trends in endocrinology and metabolism: TEM 2003;14(5):228-35.

27. Stromsoe A, Svensson L, Claesson A, Lindkvist J, Lundstrom A, Herlitz J. Association between population density and reported incidence, characteristics and outcome after out-of-hospital cardiac arrest in Sweden. Resuscitation 2011;82(10):1307-13.

28. Callaway CW, Schmicker R, Kampmeyer M, Powell J, Rea TD, Daya MR, et al. Receiving hospital characteristics associated with survival after out-of-hospital cardiac arrest. Resuscitation 2010;81(5):524-9.

29. Soholm H, Wachtell K, Nielsen SL, Bro-Jeppesen J, Pedersen F, Wanscher M, et al. Tertiary centres have improved survival compared to other hospitals in the Copenhagen area after out-of-hospital cardiac arrest. Resuscitation 2013;84(2):162-7.

30. Stub D, Smith K, Bray JE, Bernard S, Duffy SJ, Kaye DM. Hospital characteristics are associated with patient outcomes following outof-hospital cardiac arrest. Heart 2011;97(18):1489-94.

31. Choiniere R, Lafontaine P, Edwards AC. Distribution of cardiovascular disease risk factors by socioeconomic status among Canadian adults. CMAJ 2000;162(9 Suppl):S13-24.

32. Potvin L, Richard L, Edwards AC. Knowledge of cardiovascular disease risk factors among the Canadian population: relationships with indicators of socioeconomic status. CMAJ 2000;162(9 Suppl):S5-11.

33. Chiang WC, Ko PC, Chang AM, Chen WT, Liu SS, Huang YS, et al. Bystander-initiated CPR in an Asian metropolitan: does the socioeconomic status matter? Resuscitation 2014;85(1):53-8.

34. Reinier K, Thomas E, Andrusiek DL, Aufderheide TP, Brooks SC, Callaway CW, et al. Socioeconomic status and incidence of sudden cardiac arrest. CMAJ 2011;183(15):1705-12.

35. Nielsen N, Wetterslev J, Cronberg T, Erlinge D, Gasche Y, Hassager C, et al. Targeted temperature management at 33 degrees C versus 36 degrees C after cardiac arrest. N Engl J Med 2013;369(23):2197-206.

36. Strote JA, Maynard C, Olsufka M, Nichol G, Copass MK, Cobb LA, et al. Comparison of role of early (less than six hours) to later (more than six hours) or no cardiac catheterization after resuscitation from out-of-hospital cardiac arrest. Am J Cardiol 2012;109(4):451-4.

37. Grasner JT, Meybohm P, Caliebe A, Bottiger BW, Wnent J, Messelken M, et al. Postresuscitation care with mild therapeutic hypothermia and coronary intervention after out-of-hospital cardiopulmonary resuscitation: a prospective registry analysis. Crit Care 
2011;15(1):R61.

38. Hollenbeck RD, McPherson JA, Mooney MR, Unger BT, Patel NC, McMullan PW, Jr., et al. Early cardiac catheterization is associated with improved survival in comatose survivors of cardiac arrest without STEMI. Resuscitation 2014;85(1):88-95.

39. Bro-Jeppesen J, Kjaergaard J, Wanscher M, Pedersen F, Holmvang L, Lippert FK, et al. Emergency coronary angiography in comatose cardiac arrest patients: do real-life experiences support the guidelines? Eur Heart J Acute Cardiovasc Care 2012;1(4):291-301.

40. Coppler PJ, Rittenberger JC, Wallace DJ, Callaway CW, Elmer J, Pittsburgh Post Cardiac Arrest S. Billing diagnoses do not accurately identify out-of-hospital cardiac arrest patients: An analysis of a regional healthcare system. Resuscitation 2016;98:9-14. 